\title{
Achieving Optimal Throughput in Cooperative Wireless Multihop Networks with Rate Adaptation and Continuous Power Control
}

\author{
Samat Shabdanov, Patrick Mitran, Senior Member, IEEE, and Catherine Rosenberg, Fellow, IEEE
}

\begin{abstract}
This work is an offline study to characterize the performance of cooperative relaying in interference-limited multihop networks where nodes are equipped with multi-rate and continuous power control capabilities. We formulate a crosslayer flow-based framework to obtain the achievable throughput rates by jointly optimizing the parameters for multi-path routing, scheduling, rates, transmit powers and selection of cooperative nodes. This framework is generic in that it is not restricted to any particular cooperative combining technique or type of network architecture. To take continuous power control into account, we introduce a non-trivial power allocation subproblem while keeping the main cross-layer framework as a linear program. We solve the problem optimally to obtain the max-min throughput for the case when cooperation is based on the distributed Alamouti code and networks have a mesh-like topology. We derive a number of practical engineering insights based on our numerical optimal results obtained for small to medium size random networks. In particular, we establish that the use of cooperative relaying in a small to medium size random mesh network often does not yield significant performance gains in throughput and connectivity even when multi-rate and continuous power control capabilities are available at the nodes.
\end{abstract}

Index Terms-Multipoint-to-point communication, distributed Alamouti code, interference management, continuous power control, resource allocation, cooperative relaying

\section{INTRODUCTION}

Cooperative communication has recently emerged as a new paradigm in wireless networks. In conventional networks, data can only be delivered from the source to the destination over point-to-point links. However, the exclusive use of point-topoint links with single antennas at transmitters and receivers does not fully exploit the potential benefits of the wireless medium such as spatial diversity. Cooperative relaying, first proposed in [1] and [2], can exploit spatial diversity by allowing nodes to create virtual multipoint-to-point links. These virtual links are formed by a group of cooperating nodes that use their single antennas for the joint relaying of data to a common destination. We focus on fixed broadband networks with a scheduling-based access scheme. An example of such

Manuscript received June 18, 2013; revised Nov. 18, 2013 and March 20, 2014; accepted May 10, 2014. The associate editor coordinating the review of this manuscript and approving it for publication was Prof. Tommaso Melodia. The material in this paper was presented in part at the IEEE Global Communications Conference, Anaheim, CA, Dec. 2012.

S. Shabdanov, P. Mitran and C. Rosenberg are with the Dept. of Electrical and Computer Engineering, University of Waterloo, 200 University Ave. West, Waterloo, Ontario, Canada, (email: \{sshabdan,pmitran,cath\}@uwaterloo.ca).

This work was supported by the Natural Sciences and Engineering Research Council of Canada (NSERC) and Blackberry. networks is a wireless backhaul network, where base stations are connected in a mesh-like topology.

So far, very little is known about the end-to-end performance gains that can be achieved in cooperative multihop networks especially when rate adaptation and Continuous Power Control (CPC) are enabled at all nodes. Many prior studies on cooperative relaying have focused principally on the rates that can be achieved in 2-hop networks using only one source-destination pair [3], [4]. Certainly, these models cannot be used to study cooperative relaying in realistic networks where it is necessary to consider a joint configuration of many other parameters such as multiple source-destination pairs, multihop routing (with potentially more than 2 hops), power control, rate adaptation, spatial reuse and scheduling. The importance of joint configuration was previously established in [5] and [6] where it was shown that the separate optimization of some of these parameters (i.e, routing and scheduling) may not achieve optimal performance. While there exist a number of works that address the performance gains of cooperative relaying using the cross-layer approach, these works are based on simplified models or restricted cases. For instance, [7] and [8] do not consider spatial reuse and restrict the use of cooperative relaying to only a fixed selection of nodes. The study [9] is based on a simple protocol interference model, the use of which is questioned in [10], and [11] does not consider co-channel interference. In contrast with previous works, we propose a framework that is not restricted to: i) a fixed selection of cooperative relaying nodes with which to form virtual antenna arrays; ii) a simplified interference model; iii) only a few configuration parameters; or iv) discrete power control.

The objective of this work is offline study to characterize how the use of cooperative relaying impacts key network performance metrics such as throughput and connectivity. In particular, we quantify the gains that can be achieved by using continuous power control and rate adaptation in a cooperative network (as well as in a conventional one). To the best of our knowledge, this is the first work that incorporates all physical parameters (rates, powers, cooperation) and network layer parameters (routing, scheduling) under a unified framework. Importantly, we do not impose any restrictions on the interference model, selection of cooperative relaying nodes, spatial reuse, power control, or type of cooperative combining technique used to create virtual antenna arrays. This full level of parameter coupling and optimization is an important aspect of our work as only by using such a cross-layered model 
are we able to establish conclusively important engineering insights. Our framework is generic in the sense that it can be used to obtain a throughput-optimal configuration of the network that employs any type of cooperative combining technique or system architecture such as heterogeneous (e.g., [12]), ad-hoc, etc. In this paper, we extend our previous work [13] by allowing each node in the network to use rate adaptation and CPC.

We solve the problem optimally for small to medium size networks by using the column generation method. The number of variables in this problem grows exponentially with the size of the network. While the computation aspect of column generation is indeed an issue for obtaining results in large size networks, we do not address this issue in the paper but rather focus on an offline performance evaluation of cooperative relaying. Note that unlike for online solutions, there is no strict computation time requirements for offline solutions since the results can be obtained beforehand. We use column generation because this method was shown by [14] to be efficient for solving joint routing and scheduling problems. The results that we obtain using this offline study are valuable since they provide important insights to the network operators about the gain that can be obtained using cooperative relaying in their networks.

The main contributions can be summarized as follows:

- We formulate a cross-layer optimization framework that is generic and based on a realistic SINR-based interference model. This is the first work that configures cooperative relaying parameters jointly with spatial reuse, scheduling, rates, transmit powers, and routing.

- We propose an elegant approach, based on the notion of virtual nodes, to incorporate cooperative relaying into a flow-based framework. Also, while keeping the main framework as a linear program, we introduce a non-trivial power allocation subproblem to allow the use of CPC with per node power constraint.

- We solve the problem optimally when cooperative relaying is based on the distributed Alamouti code and quantify the gains in throughput and connectivity for mesh-like networks of medium size. The solutions to the problem provide us with the maximum achievable throughputs as well as the jointly-optimal network configuration for routing, scheduling, transmit power levels and the selection of cooperative relaying nodes.

- We provide a number of important engineering insights for cooperative relaying based on the Alamouti code. In particular, we show that contrary to popular belief, the use of cooperative relaying in small to medium sized random networks often provides only marginal gains in throughput as well as connectivity. We determine that the use of multiple rates or CPC yields only small performance gains in random cooperative networks. We also establish that $\mathrm{CPC}$ is an effective technique to manage interference in conventional multihop networks.

The remainder of the paper is organized as follows. Section II presents a literature review and the motivations for this work. In Section III, we describe our system model and formulate an optimization framework. In Section IV, we adapt the problem for cooperative relaying based on the Alamouti code and present engineering insights for the case of mesh networks. Section V concludes this paper.

\section{Fundamentals}

\section{A. Related work}

The concept of cooperative relaying whereby virtual antenna arrays are created was first proposed by [1] and [2] for amplify-and-forward and decode-and-forward strategies. Information-theoretic bounds to cooperative relaying have been considered in many works but these works mostly address the three-terminal channel model or networks with only one source and destination pair. Notably, the work of [5] was among the first to propose a flow-based framework that incorporates the SINR interference model. Using this framework we can find the optimal throughput rates between any number of source and destination pairs in a multihop network. Later, [6] extended the work of [5] by formulating a joint routing and scheduling problem for networks with rate adaptation and discrete power control. A similar problem was formulated in [15] but using an alternative approach based on link capacity matrices. In [14], the authors provided an offline study to evaluate the performance of rate adaptation and discrete power control in larger mesh networks. For this, the authors solved the problem in [6] using a column generation method. A main limitation of previous models in [6] and [15] is the restricted use of power control from only a finite set of discretized power levels. In this work, we improve and extend the modeling of power control by allowing nodes to choose their transmit power levels from a continuous range.

There exist a number of prior studies that address crosslayer design with cooperative relaying. However, most of these works use simplified interference models or restricted cases by limiting the number of hops, source-destination pairs or network topology. For instance, the authors of [16] computed an optimal outage probability in cooperative networks for a specific string-based topology but considered cooperative relaying only with routing. The problem of joint allocation of multi-channel transmit power levels, rates, and optimal relay node selection was studied in [17] but the model was restricted to only 2 -hop routing and interference was not considered. The works of [7] and [8] studied cooperative relaying with only a fixed selection of nodes and did not consider spatial reuse. Similar restrictions were used in [18] to optimize the average outage probability for a fixed number of hops and selection of routing paths. A distributed cooperative relaying approach was proposed by [9] but under a simple protocol interference model. Similar work was done in [11] where cooperative relaying was considered with multiple orthogonal channels and thus, the co-channel interference was not included. A distributed approach was used in [19] where cooperative relaying was jointly optimized with power control and channel allocation. While the work of [19] is not restricted to a fixed selection of cooperative relaying nodes, the model is based on an approximate interference model, restricted to singlehop routing and does not include rate adaptation. Also, the 


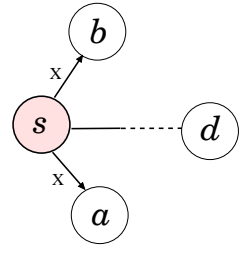

(a) Step 1,

broadcasting phase

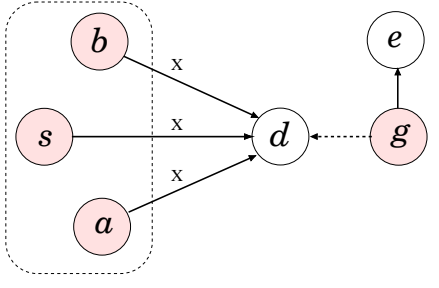

(b) Step 2,

cooperative phase
Fig. 1. Example of 3-node cooperation

problem is formulated as a non-convex problem but the authors present efficient techniques to solve it. Other works like [20] use a distributed approach based on game theory to solve a joint relay selection and power control problem but the model does not include interference. The use of cooperative relaying with power control was also studied in cognitive networks, [21], but only for a three-terminal network. Our early work [13] was among the first to study cooperative relaying jointly with multi-path routing, scheduling, and spatial reuse under a physical SINR-based interference model. It was assumed that each node used the same transmit power and same unique rate. By formulating the flow-based cross-layer framework as a linear program and optimally solving it, we quantified the end-to-end performance gains that can be achieved in wireless mesh networks with cooperative relaying. It was established in [13] that in single-rate and single-power networks, cooperative relaying does not always provide a gain in throughput or connectivity and if an improvement can be achieved then the gain is only marginal.

\section{B. Background and Motivation}

The use of multiple antennas allows a wireless network to exploit spatial diversity, improve connectivity or provide multiplexing gain. Multiple antennas can be employed either at each node or distributively at separate nodes in the network. The distributed approach implies forming a virtual antenna array where a group of nodes are able to share their individual antennas and possibly other resources for cooperative transmission. In scheduling-based networks, this can be accomplished by transmitting signals from cooperating nodes either over orthogonal channels or on the same channel by employing cooperative combining techniques.

We are interested to understand how cooperation between nodes can improve end-to-end network performance in a single-channel network. We refer to Fig. 1 as a motivational example for this paper. Let us assume that node $s$ has a message $X$ to convey to node $d$ but cannot deliver this message directly due to the large distance from $s$ to $d$. While nodes $a$ and $b$ are able to receive the message $X$ from $s$, none of these nodes alone can directly relay the information to $d$ due to poor channel conditions. Thus, using only point-to-point multihop communication, node $d$ cannot successfully receive message $X$ from node $s$. However, it might be possible for node $s$ to deliver the information with the use of cooperative relaying.

Assuming a time-slotted system, during a first slot, node $s$ broadcasts message $X$ to both nodes $a$ and $b$ as depicted in
Fig. 1(a). During a second slot, as shown in Fig. 1(b), nodes $a, b$ and $s$ can now cooperatively use the channel to transmit the same message $X$ to node $d$. By combining the signals from nodes $a, b$ and $s$, the receiver at node $d$ can now benefit from the increased signal power to decode message $X$. As a result, if this increase in signal power with respect to the interference is sufficient then node $d$ can now successfully decode message $X$. In Fig. 1(b), it is assumed that the cumulative interference at node $e$ from transmitting nodes $a, b$ and $s$ is negligible. For an optimal configuration, we must be mindful of this increased interference.

At node $d$ the signals can be spatially combined using standard techniques, such as e.g., beamforming [2] or distributed space-time block codes [1]. Both these techniques require nodes $a, b$ and $s$ to transmit simultaneously with the same rate a common message. While beamforming can be used for cooperation between any number of nodes, this technique requires perfect channel phase knowledge at the transmitters to synchronize the phases such that signals from $a, b$ and $s$ can add coherently at $d$. Space-time codes do not require perfect channel knowledge at the transmitters, but among all classes of codes only the Alamouti code can provide particularly fullrate. The use of other space-time codes results in rate loss and requires complex decoding techniques at the receiver. In the next section, we will provide a framework that can be used for any type of cooperative combining technique and will study the case of 2-node cooperation when using the distributed Alamouti code.

This example shows that cooperative relaying needs additional resource allocation since it requires now two time slots to convey message $X$ from node $s$ to $d$. Besides, cooperative transmission induces an increase in interference in the network as two or more nodes need to transmit simultaneously during the cooperative phase. As a consequence, it can potentially reduce spatial reuse of the channel. On the other hand, cooperative transmission increases the received signal power of a node which can lessen the impact of interference from other nodes transmitting on the same channel, i.e., in Fig. 1(b), node $g$ is allowed to transmit concurrently with cooperating nodes $a, b$ and $s$.

From this example in Fig. 1, we can see that cooperation may improve network performance by providing better connectivity and interference mitigation, yet there may be a price to pay in terms of spatial reuse and resource allocation. Thus, techniques that can help to manage the additional interference generated by cooperative relaying may be desirable. While there are techniques that can manage interference such as successive interference cancellation or dirty-paper coding [15], [22], the implementation of these techniques in conjunction with cooperative relaying can be difficult to accomplish. On the other hand, CPC is a very simple and efficient technique that can manage the level of interference by carefully controlling the transmit power levels at nodes during cooperative transmission.

We aim to study the impact of CPC on the performance of cooperative relaying. We also aim to study if cooperative relaying can be useful in multi-rate networks where an additional increase in signal power can be utilized to use higher 
transmission rates.

\section{OPTIMIZATION FRAMEWORK}

\section{A. Assumptions and Definitions}

Let us consider a single-channel backhaul multihop network that is comprised of a set of physical nodes $\mathcal{N}$. Each node in $\mathcal{N}$ is equipped with a single omni-directional half-duplex antenna. All physical nodes in the network are fixed and their antennas are located about $20 \mathrm{~m}$ above the ground (so that it is reasonable to assume that the channel gains are known and quasi static). If the level of co-channel interference permits, the common channel can be spatially reused by several nodes at a time. Each node in the network can support a finite set of available rates $\mathcal{R}$ and can transmit at any power level in the interval $\left[0, P_{\max }\right]$, where $P_{\max }$ is the maximum transmit power budget. Each data rate $r$ in $\mathcal{R}$ is associated with a certain SNR threshold $\beta(r)$. The choice of values for rate $r$ and threshold $\beta(r)$ depends on the modulation/coding schemes used in the network. We assume that all nodes are of the same type, and flows are given and greedy. Each node in the network can act as a source of data as well as a decode-and-forward relay. End-users (and their mobility) are not modeled in this work.

We define $k$ as a cooperation index in the network such that $k \in \mathbb{N}$ and $0<k<|\mathcal{N}|$. Index $k$ indicates the maximum number of physical nodes that are allowed to create a virtual antenna array. For example, a non-cooperative (conventional) multihop network has an index of $k=1$.

We call a virtual node the set of physical nodes that can collaboratively transmit a common message to the same destination. Virtual nodes are the groups of physical nodes that are used to model cooperative relaying as described later.

We denote by $\mathcal{V}_{k}$ the set of virtual nodes in a network with cooperative index $k$. In general, cooperative relaying is possible between any number of up to $k$ distinct physical nodes. For this reason, set $\mathcal{V}_{k}$ must include all possible combinations of up to $k$ nodes, i.e.,

$$
\mathcal{V}_{k}=\{v: v \in \mathcal{P}(\mathcal{N}) ; 0<|v| \leq k\}
$$

where $\mathcal{P}(\mathcal{N})$ is the power set of $\mathcal{N}$. Note that we define $\mathcal{V}_{k}$ to include all physical nodes in the network so that $\mathcal{N} \subset \mathcal{V}_{k}$ when $k \geq 2$. When virtual node $v$ is defined by a singleton, then $v=\{a\}$ corresponds to the physical node $a \in \mathcal{N}$. When virtual node $v$ contains $|v| \geq 2$ elements then $v$ corresponds to the set of $|v|$ physical nodes that collaborate to relay the message. We will use the notion of virtual nodes to model the conservation of information flows over communication links.

We define an information flow $f$ by the ordered pair of nodes $f=(o(f), d(f))$ and $o(f) \neq d(f)$, where $o(f)$ and $d(f)$ are the origin and the destination of flow $f$, respectively. We denote by $\mathcal{F}$ the given set of flows. Each flow $f$ in $\mathcal{F}$ can only originate at a physical node and be destined to a physical node so that $o(f) \in \mathcal{N}$ and $d(f) \in \mathcal{N}$. Let us define a communication link $\ell$ by a triple $(\mathcal{O}(\ell), \mathcal{D}(\ell), r(\ell))$, where $\mathcal{O}(\ell) \in \mathcal{V}_{k}$ and $\mathcal{D}(\ell) \in \mathcal{V}_{k}$ are the origin and destination nodes of link $\ell$, and $r(\ell) \in \mathcal{R}$ is the link data rate. The nodes $\mathcal{O}(\ell)$ and $\mathcal{D}(\ell)$ denote the set of transmitting and receiving nodes of

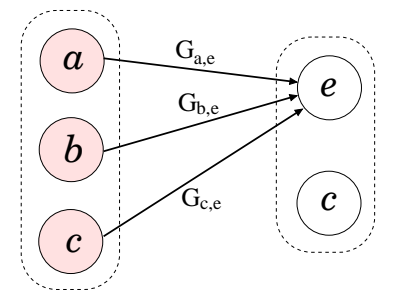

Fig. 2. Illustration of link $\ell$ defined between virtual nodes $\mathcal{O}(\ell)=\{a, b, c\}$ and $\mathcal{D}(\ell)=\{e, c\}$

link $\ell$, respectively. Later in this subsection, we will provide an example to illustrate how links with virtual nodes are used.

We denote by $P_{i}$ the transmit power of physical node $i$ where $P_{i} \in\left[0, P_{\text {max }}\right]$ and by $\mathbf{P}_{\mathcal{O}(\ell)}=\left[P_{i}\right]_{i \in \mathcal{O}(\ell)}$ the vector of transmit powers of all the physical nodes in $\mathcal{O}(\ell)$. We also define $F_{\text {sig }}\left(\mathbf{P}_{\mathcal{O}(\ell)}, \mathbf{G}_{\mathcal{O}(\ell), j}\right)$ to be the received signal power at node $j$ as a function of $\mathbf{P}_{\mathcal{O}(\ell)}$ and the channel power gains $\mathbf{G}_{\mathcal{O}(\ell), j}=\left[G_{i, j}\right]_{i \in \mathcal{O}(\ell)}$ from nodes in $\mathcal{O}(\ell)$ to node $j \in \mathcal{D}(\ell)$. The function $F_{\text {sig }}(\cdot)$ is a general function that represents the type of cooperative combining technique used in the network, e.g., in the case of a technique where transmitters use independent codebooks, $F_{\text {sig }}(\cdot)$ is

$$
F_{\text {sig }}\left(\mathbf{P}_{\mathcal{O}(\ell)}, \mathbf{G}_{\mathcal{O}(\ell), j}\right)=\sum_{i \in \mathcal{O}(\ell)} P_{i} G_{i, j}
$$

The channel gain $G_{i, j}$ between physical nodes $i$ and $j$ is modeled as

$$
G_{i, j}=P L\left(d_{i, j}\right) \cdot g_{i, j}
$$

i.e., it is the aggregation of the fading power gain $g_{i, j}$ and the path loss $P L\left(d_{i, j}\right)$ at distance $d_{i, j}$.

While a link can be defined between any pair of virtual nodes, not all links are feasible. A link $\ell$ is called feasible to support the link rate $r(\ell)$ if, in the absence of interference, the following holds:

$$
\begin{aligned}
& {[\mathbf{C 1}] \quad \mathcal{D}(\ell) \backslash \mathcal{O}(\ell) \neq \emptyset ;} \\
& {[\mathbf{C 2}] \quad \forall j \in \mathcal{D}(\ell) \backslash \mathcal{O}(\ell): \frac{F_{\text {sig }}\left(\mathbf{P}_{\mathcal{O}(\ell)}^{\max }, \mathbf{G}_{\mathcal{O}(\ell), j}\right)}{N_{0}} \geq \beta(r(\ell)),}
\end{aligned}
$$

where $\mathbf{P}_{\mathcal{O}(\ell)}^{\max }=\left[P_{\max }\right]_{i \in \mathcal{O}(\ell)}$. Condition [C1] states that any feasible link $\ell$ must have at least one physical node in $\mathcal{D}(\ell)$ that is not in $\mathcal{O}(\ell)$. If all nodes in $\mathcal{D}(\ell)$ are in $\mathcal{O}(\ell)$, then link $\ell$ is meaningless in the sense that all physical nodes in $\mathcal{D}(\ell)$ already have the message intended for transmission from $\mathcal{O}(\ell)$.

The LHS of condition [C2] is the SNR at node $j$, where $N_{0}$ is the thermal noise and $F_{\text {sig }}\left(\mathbf{P}_{\mathcal{O}(\ell)}^{\max }, \mathbf{G}_{\mathcal{O}(\ell), j}\right)$ is the signal power at the receiver of node $j$ when all nodes in $\mathcal{O}(\ell)$ transmit with the maximum power $P_{\max }$. For link $\ell$ to be feasible, a message transmitted cooperatively by the nodes in $\mathcal{O}(\ell)$ must be successfully decoded by all nodes in $\mathcal{D}(\ell)$ that are not in $\mathcal{O}(\ell)$. For successful decoding of a message, the SNR at each node $j$ in $\mathcal{D}(\ell) \backslash \mathcal{O}(\ell)$ must meet the minimum threshold $\beta(r(\ell))$ for the rate $r(\ell)$.

We denote by $\mathcal{L}_{k}$ the set of feasible links in the network with index $k$ and set of virtual nodes $\mathcal{V}_{k}$. The set of feasible 
a)

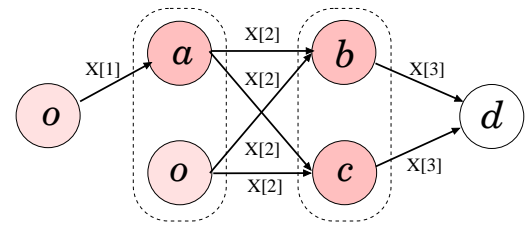

b) $\quad v_{1} \stackrel{\mathrm{X}[1]}{\longrightarrow} v_{2} \stackrel{\mathrm{X}[2]}{\longrightarrow} v_{3} \stackrel{\mathrm{X}[3]}{\longrightarrow} v_{4}$

Fig. 3. a) 3-hop transmission of message $X$ from node $s$ to $d$; b) equivalent representation for routing using virtual nodes

links is defined as follows:

$$
\begin{array}{r}
\mathcal{L}_{k}=\left\{\ell=(\mathcal{O}(\ell), \mathcal{D}(\ell), r(\ell)): \mathcal{O}(\ell), \mathcal{D}(\ell) \in \mathcal{V}_{k} ;\right. \\
r(\ell) \in \mathcal{R} ; \ell \text { meets }[\mathbf{C 1}] \text { and }[\mathbf{C 2}]\} .
\end{array}
$$

To illustrate the use of virtual nodes for modeling feasible links, let us consider the example in Fig. 2. It shows the multipoint-to-point link $\ell$ that is defined between virtual nodes $\mathcal{O}(\ell)=\{a, b, c\}$ and $\mathcal{D}(\ell)=\{e, c\}$, i.e., a group of nodes establishes a transmission to each physical node in $\mathcal{D}(\ell)$. This link is feasible to support the rate $r(\ell)$ if node $e$ obeys condition [C2] for a given signal combining function $F_{\text {sig }}(\cdot)$ and the vector of channel power gains $\mathbf{G}_{\mathcal{O}(\ell), e}=\left[G_{a, e}, G_{b, e}, G_{c, e}\right]^{T}$. Node $c$ does not need to meet [C2] since this node is in $\mathcal{O}(\ell)$ and already has the message intended for transmission from nodes $\{a, b, c\}$. With this example, we also show that the origin and destination of links may have common physical nodes, i.e., node $c$ is shared between $\mathcal{O}(\ell)$ and $\mathcal{D}(\ell)$. While these common physical nodes do not participate in all transmissions of data, they are used to model flow conservation. Fig. 3 shows an example of a cooperative network where source node $o$ delivers message $X$ to destination $d$ using feasible links. During the first hop, the message is transmitted from virtual node $v_{1}=\{o\}$ to $v_{2}=\{a, o\}$, during the second hop, node $v_{2}=\{a, o\}$ transmits it to node $v_{3}=\{b, c\}$, and during the last hop, message $X$ is delivered to destination node $v_{4}=\{d\}$ from virtual node $v_{3}$. Fig. 3 also shows an equivalent routing model to illustrate that the information must be conserved at each virtual node along the routing path. The notion of feasible links defined in $[\mathbf{C 1}],[\mathbf{C 2}]$ allows us to formulate a framework for throughput optimization of a network that employs any type of cooperative combining technique.

In the following, we model a wireless network with cooperative index $k$ as a set of virtual nodes $\mathcal{V}_{k}$, a set of flows $\mathcal{F}$ and a set of feasible links $\mathcal{L}_{k}$.

\section{B. Conflict-free Scheduling and ISets}

We define a conflict-free schedule as a schedule that activates at each given time a set of links that do not cause harmful interference to any receivers, i.e., all the corresponding receivers can successfully decode the signals that are intended for them. We define an ISet (for independent set) $s$ as a set of links that can transmit concurrently without causing harmful interference to each receiver. By activating only ISets, the scheduling is guaranteed to be conflict-free [6]. Clearly, for a set of links $s$ to be an ISet, it must meet certain conditions that will be presented later in this subsection. Note that an ISet specifies not only the set of links that are active but also the corresponding set of rates.

We denote by $\mathbf{P}_{\mathcal{O}(s)}=\left[\mathbf{P}_{\mathcal{O}(\ell)}\right]_{\ell \in s}$ the vector of transmit powers of all the physical nodes that transmit in ISet $s$. We denote by $\alpha_{s}$ the fraction of time ISet $s$ is activated for transmission with power vector $\mathbf{P}_{\mathcal{O}(s)}$, i.e. an ISet $s$ is scheduled if $\alpha_{s}>0$, otherwise $\alpha_{s}=0$. In practice, a conflict-free schedule is an assignment of Spatial Time Division Multiple Access (STDMA) slots to ISets. A STDMA frame is the schedule cycle that consists of a sequence of STDMA slots during which ISets are activated for transmission. A STDMA frame must be known by all nodes in a network. Clearly, for the efficient utilization of the channel, the STDMA frame must be optimally configured. Usually, the STDMA frame is optimally configured by solving a binary program similar to [23]. To overcome the complexity of integer programming, we assume the STDMA frame to be infinitely long to allow a fractional and flow-based model similar to [5], [6], [14]. A conflict-free schedule is characterized by the vector $\boldsymbol{\alpha}=\left[\alpha_{s}\right]_{s \in \mathcal{I}_{k}}$. Since, $\alpha_{s}$ is a fraction of time in one schedule cycle (STDMA frame) then $\sum_{s \in \mathcal{I}_{k}} \alpha_{s}=1$.

Multiple rates and single power: First let us define ISets for networks where nodes can support a set of discrete rates $\mathcal{R}$ but are allowed to transmit only with a single power, i.e., the maximum power $P_{\max }$ and thus $\mathbf{P}_{\mathcal{O}(s)}=\left[P_{\mathcal{O}(\ell)}^{\max }\right]_{\ell \in s}$. A set of links $s \subseteq \mathcal{L}_{k}$ is an ISet if it obeys the following conditions:

[C3] for all $\ell_{1}, \ell_{2} \in s, \ell_{1} \neq \ell_{2}: \mathcal{O}\left(\ell_{1}\right) \cap \mathcal{O}\left(\ell_{2}\right)=\emptyset$;

[C4] for all $\ell_{1}, \ell_{2} \in s, \ell_{1} \neq \ell_{2}: \mathcal{O}\left(\ell_{1}\right) \cap \mathcal{D}\left(\ell_{2}\right)=\emptyset$;

[C5] for all $\ell_{1}, \ell_{2} \in s, \ell_{1} \neq \ell_{2}: \mathcal{D}\left(\ell_{1}\right) \cap \mathcal{D}\left(\ell_{2}\right)=\emptyset$;

[C6] for all $\ell \in s: \forall j \in \mathcal{D}(\ell) \backslash \mathcal{O}(\ell)$

$$
\frac{F_{\text {sig }}\left(\mathbf{P}_{\mathcal{O}(\ell)}, \mathbf{G}_{\mathcal{O}(\ell), j}\right)}{N_{0}+F_{\text {int }}\left(\mathbf{P}_{\mathcal{O}(s \backslash \ell)}, \mathbf{G}_{\mathcal{O}(s \backslash \ell), j}\right)} \geq \beta(r(\ell)) .
$$

Conditions [C3] - [C5] are the half-duplex requirements and state that no two distinct links in ISet $s$ can share a physical node of origin or destination.

The LHS of condition [C6] is the SINR at the receiver of node $j$. Each node in $\mathcal{D}(\ell) \backslash \mathcal{O}(\ell)$ must meet the SINR threshold $\beta(r(\ell))$ to allow error-free transmission of data at rate $r(\ell)$ over link $\ell$. In condition [C6], $F_{\text {int }}\left(\mathbf{P}_{\mathcal{O}(s \backslash \ell)}, \mathbf{G}_{\mathcal{O}(s \backslash \ell), j}\right)$ is the interference power at node $j$ that is a function of the transmit power $\mathbf{P}_{\mathcal{O}(s \backslash \ell)}=\left[P_{i}\right]_{i \in \mathcal{O}\left(\ell^{\prime}\right) \ell^{\prime} \in s \backslash \ell}$ and the channel gains $\mathbf{G}_{\mathcal{O}(s \backslash \ell), j}=\left[G_{i, j}\right]_{i \in \mathcal{O}\left(\ell^{\prime}\right) ; \ell^{\prime} \in s \backslash \ell}$ vectors from all the interfering nodes that are in the ISet $s$ but not in $O(\ell)$. The function $F_{\text {int }}(\cdot)$ for the interference power may differ from the signal combining function $F_{\text {sig }}(\cdot)$. For instance in the case of distributed beamforming, transmitters use dependent codebooks for transmission of in-phase signals that are unlikely to arrive as in-phase interfering signals at any node in the network. On other hand, when transmitters use independent codebooks, the interference power at node $j \in \mathcal{D}(\ell)$ is then

$$
F_{\text {int }}\left(\mathbf{P}_{\mathcal{O}(s \backslash \ell)}, \mathbf{G}_{\mathcal{O}(s \backslash \ell), j}\right)=\sum_{\substack{\ell^{\prime} \in s \backslash \ell \\ i \in \mathcal{O}\left(\ell^{\prime}\right)}} P_{i} G_{i, j} .
$$


Let us denote by $\mathcal{I}_{S P, k}$ the set of all ISets in the singlepower network with index $k$ that is defined as follows:

$$
\mathcal{I}_{S P, k}=\left\{s: s \in \mathcal{P}\left(\mathcal{L}_{k}\right) ;|s|>0 ; s \text { meets }[\mathbf{C 3}]-[\mathbf{C 6}]\right\},
$$

where $\mathcal{P}\left(\mathcal{L}_{k}\right)$ is the power set of the set of feasible links $\mathcal{L}_{k}$.

Multiple rates and continuous power control: Now we define ISets for networks where nodes use power control. In comparison with previous works [6], [15], where power control is used with discretized power levels only, we allow nodes to adjust their transmit powers in a continuous range.

A set of links $s \subseteq \mathcal{L}_{k}$ is an ISet in a network with CPC if $s$ obeys conditions [C3]-[C5] and in addition, each link $\ell$ in $s$ meets the SINR threshold $\beta(r(\ell))$ for a certain feasible allocation of powers for $\mathbf{P}_{\mathcal{O}(s)}$. The solution for $\mathbf{P}_{\mathcal{O}(s)}$ is not necessarily unique but since we do not have any restrictions on the allocation of transmit powers, we can just check if there exists at least one solution for $\mathbf{P}_{\mathcal{O}(s)}$ in the feasible region. A power allocation subproblem to check if there exist any feasible solution for $\mathbf{P}_{\mathcal{O}(s)}$ can be formulated as follows:

[C7] there exists a feasible $\mathbf{P}_{\mathcal{O}(s)}$ if $\Delta=0$ is the solution to the subproblem:

$$
\begin{gathered}
\Delta=\min _{\mathbf{P}_{\mathcal{O}(s)}} \sum_{\substack{\ell \in s \\
j \in \mathcal{D}(\ell) \backslash \mathcal{O}(\ell)}} \phi_{\ell, j} \\
\text { such that: } \\
F_{\text {sig }}\left(\mathbf{P}_{\mathcal{O}(\ell)}, \mathbf{G}_{\mathcal{O}(\ell), j}\right)-\beta(r(\ell)) N_{0} \\
-F_{\text {int }}\left(\mathbf{P}_{\mathcal{O}(s \backslash \ell)}, \mathbf{G}_{\mathcal{O}(s \backslash \ell), j}\right) \beta(r(\ell))+\phi_{\ell, j} \geq 0 \quad \begin{array}{r}
\forall \ell \in s \\
\forall j \in \mathcal{D}(\ell) \backslash \mathcal{O}(\ell)
\end{array} \\
\boldsymbol{\phi}, \mathbf{P}_{\mathcal{O}(s)} \geq 0 \\
0 \leq \mathbf{P}_{\mathcal{O}(s)} \leq P_{\max }
\end{gathered}
$$

The subproblem in [C7] is a linear program similar to the problem described in [22]. We denote by $\phi=\left[\phi_{\ell, j}\right]_{\ell \in s ; j \in \mathcal{D}(\ell) \backslash \mathcal{O}(\ell)}$ the vector of indicator variables for constraints in $[\mathbf{C 7}]$. For a set of links $s$ to be an ISet, each node in $\mathcal{D}(\ell) \backslash \mathcal{O}(\ell)$ is required to meet the threshold $\beta(r(\ell))$ for the feasible transmit power vector $\mathbf{P}_{\mathcal{O}(s)}$ over all links $\ell$ in $s$. We claim a set of links $s$ is an ISet iff $\Delta=0$. This is because $\phi \geq 0$ and since $\Delta$ is lower bounded by $\Delta \geq 0$, then $\Delta=0$ iff all $\phi_{\ell, j}=0$. Now, if all $\phi_{\ell, j}=0$ then ISet $s$ meets the condition (5) for a feasible allocation of transmit powers $\mathbf{P}_{\mathcal{O}(s)}$. If $\Delta>0$ then $s$ is not an ISet since there does not exist $\mathbf{P}_{\mathcal{O}(s)}$ that allows ISet $s$ to meet condition (5). In the next section, we will show how to use condition $[\mathbf{C 7}]$ for the case of cooperative relaying based on the Alamouti code.

We denote by $\mathcal{I}_{C P C, k}$ the collection of all ISets in the network with CPC and cooperative index $k$ that is defined as follows:

$$
\begin{aligned}
& \mathcal{I}_{C P C, k}=\left\{s: s \in \mathcal{P}\left(\mathcal{L}_{k}\right) ;|s|>0\right. \\
&s \text { meets }[\mathbf{C 3}]-[\mathbf{C 5}] \text { and }[\mathbf{C 7}]\},
\end{aligned}
$$

where $\mathcal{P}\left(\mathcal{L}_{k}\right)$ is the power set of $\mathcal{L}_{k}$. We associate with each ISet $s \in \mathcal{I}_{C P C, k}$ the transmit power vector $\mathbf{P}_{\mathcal{O}(s)}$ that is obtained by solving the subproblem in [C7].

\section{Problem Formulation}

In the following, we formulate a flow-based problem to find the optimal flow rates in a cooperative network with index $k$ and for the given sets $\mathcal{L}_{k}, \mathcal{F}, \mathcal{V}_{k}$ and $\mathcal{I}_{k}$. We adapt the framework of [6] as opposed to the one developed in [15] because the latter: i) does not allow multi-path routing; and ii) is difficult to obtain the results by checking the feasibility of the capacity region matrix.

Let us denote by $x_{f}(\ell)$ the amount of flow $f$ transmitted over link $\ell \in \mathcal{L}_{k}$ and by $R_{f}$ the throughput rate of flow $f$. Also, we denote by $\boldsymbol{x}=\left[x_{f}(\ell)\right]_{\ell, f}$ the aggregated routing vector of all flows allocated over all feasible links and by $\boldsymbol{R}=\left[R_{f}\right]_{f \in \mathcal{F}}$ the vector of achievable flow rates. While the parameters for multihop and multi-path routing are represented in $x$, the parameters for spatial reuse, rate adaptation and power control are incorporated in the sets $\mathcal{L}_{k}$ and $\mathcal{I}_{k}$ where the set of all ISets $\mathcal{I}_{k}$ is either $\mathcal{I}_{k}=\mathcal{I}_{S P, k}$ for single-power networks or $\mathcal{I}_{k}=\mathcal{I}_{C P C, k}$ for networks with CPC. We further assume that the traffic is static or quasi-static to enable a flowbased formulation. The joint routing, scheduling, cooperative relaying, rate adaptation, and CPC problem for the max-min throughput $R$ is then formulated as follows [5], [6]:

$$
\text { [M]: } \quad \max _{\boldsymbol{\alpha}, \boldsymbol{x}} R
$$

such that:

$$
\begin{aligned}
& \sum_{\substack{\ell \in \mathcal{L}_{k} \\
\mathcal{O}(\ell)=n}} x_{f}(\ell)-\sum_{\substack{\ell \in \mathcal{L}_{k} \\
\mathcal{D}(\ell)=n}} x_{f}(\ell)=\left\{\begin{array}{cl}
R_{f}, & n=o(f) \\
-R_{f}, & n=d(f) \\
0, & \text { else }
\end{array} \quad \begin{array}{l}
\forall n \in \mathcal{V}_{k} \\
\forall f \in \mathcal{F}
\end{array}\right. \\
& r(\ell) \sum_{s \in \mathcal{I}_{k}} \alpha_{s} \mathbf{1}_{\{\ell \in s\}} \geq \sum_{f \in \mathcal{F}} x_{f}(\ell) \quad \forall \ell \in \mathcal{L}_{k} \\
& R_{f} \geq w_{f} R \quad \forall f \in \mathcal{F} \\
& \sum_{s \in \mathcal{I}_{k}} \alpha_{s}=1 \\
& \alpha, x, R \geq 0 .
\end{aligned}
$$

Constraints in (10) are the flow conservation conditions specified for each flow and virtual node, i.e., the amount of flow arriving to a virtual node must be equal to the amount of flow leaving a virtual node. Link scheduling constraints are given in (11) and (13). Specifically, constraints in (11) restrict the total amount of flow that can be scheduled over a link to its link rate capacity $r(\ell) \sum_{s \in \mathcal{I}_{k}} \alpha_{s} \mathbf{1}_{\{\ell \in s\}}$. The indicator function $\mathbf{1}_{\{\ell \in s\}}$ serves to include in the addition only those ISets that contain link $\ell$. Typically in practical wireless networks, nodes need to support flows with unequal rates. For this reason, we formulate problem $[\mathbf{M}]$ with the flow weighted constraints (12), where $w_{f}$ denotes the weight of flow $f$ with respect to $R$. Constraints (13) state that the ISets must be scheduled over a unit period of time.

We formulate $[\mathbf{M}]$ for the max-min throughput as this choice of objective function prevents the relative starvation of nodal flow rates while trying to maximize the system throughput. A solution to $[\mathbf{M}]$ provides us with the optimal throughput rates and optimal network configuration. The optimal network configuration comprises the routing $x$, scheduling $\alpha$, and transmit power $\left[\mathbf{P}_{\mathcal{O}(s)}\right]_{s \in \mathcal{I}_{k}}$ as well as the selection of cooperative 
nodes. We improve upon the previous frameworks in [6] and [15] by providing: i) an elegant approach based on the notion of virtual nodes to incorporate cooperative relaying into the joint routing and scheduling problem while keeping $[\mathbf{M}]$ as a LP; ii) the novel power allocation subproblem in [C7] to allow a non-trivial addition of CPC to the problem formulation.

\section{Problem Complexity}

In a network with $|\mathcal{N}|$ physical nodes and cooperation index $k$, the number of virtual nodes $\left|\mathcal{V}_{k}\right|$ is $\left(\begin{array}{c}|\mathcal{N}| \\ 1\end{array}\right)+\cdots+\left(\begin{array}{c}|\mathcal{N}| \\ k\end{array}\right)$. Thus, in a network with $|\mathcal{R}|$ rates and $\left|\mathcal{V}_{k}\right|$ virtual nodes, the maximum number of links and ISets are exponential in $O\left(\left|\mathcal{V}_{k}\right|^{2}|\mathcal{R}|\right)$ and $O\left(2^{\left|\mathcal{L}_{k}\right|}\right)$, respectively. In order to enumerate all possible ISets, it is necessary to check all elements in the power set of $\mathcal{L}$ for being an ISet. In addition, the subproblem in condition [C7] must be solved for each ISet. Certainly, the use exhaustive search to find all ISets is intractable even for small size networks with small index $k$ due to the fact that the size of sets $\mathcal{V}_{k}$ and $\mathcal{I}_{k}$ grow exponentially. The NP-hardness of problems of type [M] (joint routing and scheduling) was studied and proved in [5].

Instead, we use the column generation method to solve problem [M] optimally. This method allows us to avoid an exhaustive enumeration of all ISets. Since the number of constraints in (11) is significantly less than the number of variables, an optimal solution can be found using only a very small subset of ISets, i.e., at most $\left|\mathcal{L}_{k}\right|+1$ ISets are needed to obtain an optimal solution [14]. The search for ISets is performed iteratively based on their reduced costs. In the column generation method, the solution is determined to be optimal if no ISets can be found with strictly positive reduced costs. The reduced costs are computed for a new off-basis column or ISet $s$ as (see [14]): $-\left(\zeta+\sum_{\ell \in s} r(\ell) \mu_{\ell}\right)$, where $\mu_{\ell}$ and $\zeta$ are the dual variables for (11) and (13), respectively. Additional details on the column generation method as well as convergence properties can be found in the work of [14].

\section{The CASE Of CoOperation BASED ON THE Alamouti Code}

In this section, we show how to specialize the crosslayer framework in Section III for the case when cooperation between nodes is performed using the Alamouti code. We quantify the gains of this technique in mesh-like networks where nodes support CPC and rate adaptation. Note that the framework described in Section III is not restricted to meshlike networks and in general, can be used in heterogeneous or ad-hoc networks by defining accordingly the set of flows $\mathcal{F}$.

\section{A. Encoding Scheme}

In the following, we consider a network where cooperation is allowed between two nodes only, i.e., a network with cooperative index $k=2$. While, with the use of nonorthogonal Space-Time Block Codes (STBC), it is possible to achieve cooperation between any number of nodes, it is hard to accomplish in practice due to the increased complexity of decoding at the receivers [24]. In the case of a two antenna array, STBC are represented as the full-rate Distributed Alamouti Code (D-AC). It is a relatively simple technique to implement as it does not require challenging phase synchronization (compared to distributed beamforming), and nodes can be perfectly synchronized in the context of a scheduling-based network.

To illustrate the D-AC [1], let us consider an example based on Fig. 4(c). Nodes $a$ and $b$ form a virtual antenna array and intend to send a common message $\boldsymbol{X}=\left[x_{1}, x_{2}, \ldots, x_{m}\right]$ to node $d$. Let us assume that the information symbols are independent and belong to a complex constellation with unit energy and zero mean so that $x_{i} \in \mathbb{C}$ and $\mathbb{E}\left\{x_{i}\right\}=0$. Also, let channel gains $h_{1}, h_{2} \in \mathbb{C}$ remain constant during the transmission of $\boldsymbol{X}$ and be known at the receiver. Nodes $a$ and $b$ split the message $\boldsymbol{X}$ into two equal parts $\boldsymbol{X}_{1}, \boldsymbol{X}_{2}$ so that $\boldsymbol{X}_{1}=\left[x_{1}, . ., x_{m / 2}\right]^{T}$ and $\boldsymbol{X}_{2}=\left[x_{m / 2+1}, \ldots, x_{m}\right]^{T}$. During the first half of a slot, nodes $a$ and $b$ send $\boldsymbol{X}_{1}$ and $\boldsymbol{X}_{2}$, respectively. During the second half of a slot, node $a$ sends $-\boldsymbol{X}_{2}^{*}$ and node $b$ sends $\boldsymbol{X}_{1}^{*}$. If nodes $a$ and $b$ transmit with power levels $P_{1}$ and $P_{2}$, respectively, then the received signal $Y$ at node $d$ is:

$$
[\boldsymbol{Y}[1] \boldsymbol{Y}[2]]=\left[h_{1} h_{2}\right]\left[\begin{array}{cc}
\sqrt{P_{1}} \boldsymbol{X}_{1} & -\sqrt{P_{1}} \boldsymbol{X}_{2}^{*} \\
\sqrt{P_{2}} \boldsymbol{X}_{2} & \sqrt{P_{2}} \boldsymbol{X}_{1}^{*}
\end{array}\right]+[\boldsymbol{w}[1] \boldsymbol{w}[2]],
$$

where $\boldsymbol{w}[1]$ and $\boldsymbol{w}[2]$ are the vectors of additive white Gaussian noise with variance $N_{0}$ in the first and the second half of a slot, respectively. Once nodes $a$ and $b$ complete the transmission, node $d$ can start symbol-wise decoding of the transmitted symbols using the vector of estimates for $\boldsymbol{X}$ :

$$
\left[\begin{array}{c}
\hat{\boldsymbol{X}}_{1} \\
\hat{\boldsymbol{X}}_{\mathbf{2}}
\end{array}\right]=\left[\begin{array}{c}
\sqrt{P_{1}} h_{1}^{*} \boldsymbol{Y}[1]+\sqrt{P_{2}} h_{2} \boldsymbol{Y}^{*}[2] \\
-\sqrt{P_{1}} h_{1} \boldsymbol{Y}^{*}[2]+\sqrt{P_{2}} h_{2}^{*} \boldsymbol{Y}[1]
\end{array}\right] .
$$

Such an encoding scheme uses independent codebooks at the transmitters of nodes $a$ and $b$ in each half of the slot so that $\mathbb{E}\left\{x_{i} x_{j}^{*}\right\}=0$ for $i \neq j$. The SNR at the receiver of node $d$ is then $\left(P_{1} G_{1}+P_{2} G_{2}\right) / N_{0}$, where $G_{1}=\left|h_{1}\right|^{2}$ and $G_{2}=$ $\left|h_{2}\right|^{2}$ are the channel power gains. When the SNR is at least the minimum threshold $\beta(r(\ell))$ then node $d$ can successfully decode $X$ transmitted on link $\ell$ at rate $r(\ell)$, i.e., link $\ell=$ $(\{a, b\},\{d\})$ is feasible. As a result, the use of D-AC increases the received signal power at node $d$ by combining the signals from the two distributed antennas. There is no rate loss in this scheme as it takes a slot to transmit the entire message $X$.

\section{B. ISets with $D-A C$}

Consider a network with $k=2$ and a set of physical nodes $\mathcal{N}$. All nodes in the network are enabled with D-AC, rate adaptation, and CPC. The set of virtual nodes $\mathcal{V}_{2}$ is defined according to (1).

We can define the set of feasible links $\mathcal{L}_{2}$ using the definition (4). In conditions [C2] and [C6], we can use (2) to find the received signal power since with D-AC the transmitters use independent codebooks. Unlike in [7], [8], the notion of virtual nodes in (1) allows us to consider all types of point-to-multipoint links without restrictions on the selection of nodes participating in cooperative transmission. Fig. 4 illustrates the possible type of links that can exist in 


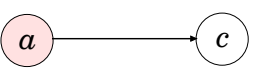

(a) $\ell=(\{a\},\{c\})$

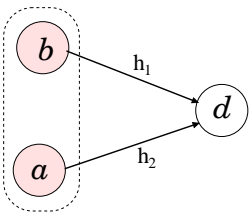

(c) $\ell=(\{a, b\},\{d\})$

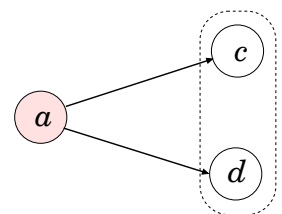

(b) $\ell=(\{a\},\{c, d\})$

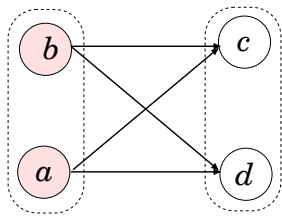

(d) $\ell=(\{a, b\},\{c, d\})$
Fig. 4. Illustration of types of links in the network with D-AC

a network with D-AC. Fig. 4(a) shows the point-to-point link between two non-cooperative nodes $\{a\}$ and $\{c\}$. Fig. 4(b) shows the link between node $\{a\}$ and cooperative node $\{c, d\}$ that models a broadcast transmission. Fig. 4(c) shows the link when the channel is cooperatively used by nodes $a$ and $b$ for transmission to node $d$. The link in Fig. 4(d) represents the transmission between two distributed source and destination nodes (distributed MIMO).

In a network with CPC, we can find the set of ISets $\mathcal{I}_{C P C, 2}$ using definition (8). In conditions [C6] and [C7], we use the function in (6) for the interference power since with D-AC, the interference is a compound signal from all interfering nodes in an ISet that transmit independently encoded messages.

To find $\mathcal{I}_{S P, 2}$ in a single-power network, we use definition (7). In condition $[\mathbf{C 6}]$ we use the functions for $F_{\text {sig }}(\cdot)$ and $F_{\text {int }}(\cdot)$ as defined in (2) and (6), respectively.

In the case of non-cooperative networks with $k=1$, we can use the same definitions to find $\mathcal{V}_{1}, \mathcal{L}_{1}, \mathcal{I}_{S P, 1}$ for single-power networks, and $\mathcal{I}_{C P C, 1}$ for networks with CPC. As nodes are not able to cooperate, then each virtual node in $\mathcal{V}_{1}$ is a physical node, i.e., $\mathcal{N}=\mathcal{V}_{1}$.

Once the sets of virtual nodes, feasible links, and ISets are properly defined, we can solve problem $[\mathbf{M}]$ to find an optimal network configuration and the achievable flow rates. In the next section, we will provide numerical results that are obtained using the column generation method discussed earlier.

\section{Numerical Results and Engineering Insights}

Parameter settings and performance metrics: We provide exact numerical solutions for single-gateway mesh networks, where nodes are fixed and all have the same transmit power budget $P_{\max }$. We assume that each node has a flow destined to the gateway (uplink flow) as well as one from the gateway (downlink flow). We consider a mesh network where each node has equal downlink to uplink flow weights, i.e., $w_{f}=1$ for all flows. Let $R$ be the max-min throughput defined in Section III-C. We call $R^{*}=2 R$ the nodal max-min throughput.

We generate an $N$-node network realization by placing $N-$ 1 nodes uniformly at random in a $2 \mathrm{~km}$ by $2 \mathrm{~km}$ square and the gateway in the center of the square. For each realization, we use (3) to model the channel power gains. As for the path loss in (3) we use the following [25]:

$$
P L\left(d_{i, j}\right)= \begin{cases}\left(\frac{\lambda}{4 \pi d}\right)^{2} & d_{i, j}<d_{0} \\ \left(\frac{\lambda}{4 \pi d_{0}}\right)^{2}\left(\frac{d_{i, j}}{d_{0}}\right)^{\mu} & d_{i, j} \geq d_{0},\end{cases}
$$

where $\mu=-3.3$ is the path loss exponent, $\lambda=0.3 \mathrm{~m}$ is the wavelength and $d_{0}=30 \mathrm{~m}$ is the reference distance of the near field. The fading gains $g_{i, j}$ are modeled as independent exponentially distributed power gains with unit mean. The thermal noise is $N_{0}=-100 \mathrm{dBm}$.

When the multi-rate case is considered, we allow a network to use rate adaptation from the set of discrete rates $\mathcal{R}=\{1,2\}$ with the corresponding thresholds $\beta(1)=3 \mathrm{~dB}$ and $\beta(2)=10 \mathrm{~dB}$. We denote by $r_{m}$ the highest rate in $\mathcal{R}$. We solve problem [M] for 100 random network realizations. Due to the complexity of problem [M], we could obtain optimal results for the case of D-AC with $\mathrm{CPC}$ and multiple rates only for mesh networks of size $N=10$ nodes (including the gateway).

We denote by JRS the results that are obtained for noncooperative networks and by $\mathrm{D}-\mathrm{AC}$ the results for cooperative networks. When CPC is enabled, the results are denoted as $\mathrm{JRS}+\mathrm{CPC}$ and D-AC+CPC for non-cooperative and cooperative networks, respectively.

We use the metric gain $\left(P_{\max }\right)$ to characterize the relative average throughput gain over 100 realizations between a scheme $A$ with respect to a scheme $B$, where

$$
\operatorname{gain}\left(P_{\max }\right)=\frac{\bar{R}_{A}\left(P_{\max }\right)-\bar{R}_{B}\left(P_{\max }\right)}{\bar{R}_{B}\left(P_{\max }\right)} \times 100 \%,
$$

where $\bar{R}_{A}\left(P_{\max }\right)$ and $\bar{R}_{B}\left(P_{\max }\right)$ denote the averaged max-min throughputs over the 100 network realizations of schemes $A$ and $B$, respectively, when nodes have power budget $P_{\text {max }}$. Note that the results for gain $\left(P_{\max }\right)$ are provided for different values of $P_{\max }$ starting at the minimum $P_{\max }$ at which a noncooperative network can be fully connected, i.e., the minimum $P_{\max }$ at which $R^{*}>0$ in the JRS results.

We define $P_{S H}$ for a given scheme as the minimum power for which the max-min nodal throughput is $r_{m} /(N-1)$ (the maximum achievable nodal throughput). We then define a second metric $\Delta P_{S H}$ as the power difference in $P_{S H}$ between two schemes.

Single rate and single power: We refer to our prior work [13] for the case when D-AC is used in networks where nodes can only transmit with the single power $P_{\max }$ and support the single rate $\mathcal{R}=\{r\}$. In [13], we established that in a mid-size mesh network, D-AC can only provide marginal gain in throughput, in $P_{S H}$, as well as in connectivity. In the following, we do not study the latter as the connectivity in a mesh network cannot be improved by the use of CPC or the addition of higher rates. This is due to the fact that the connectivity of a mesh network is determined by the node with the worst channel conditions and the minimum available rate in $\mathcal{R}$. 


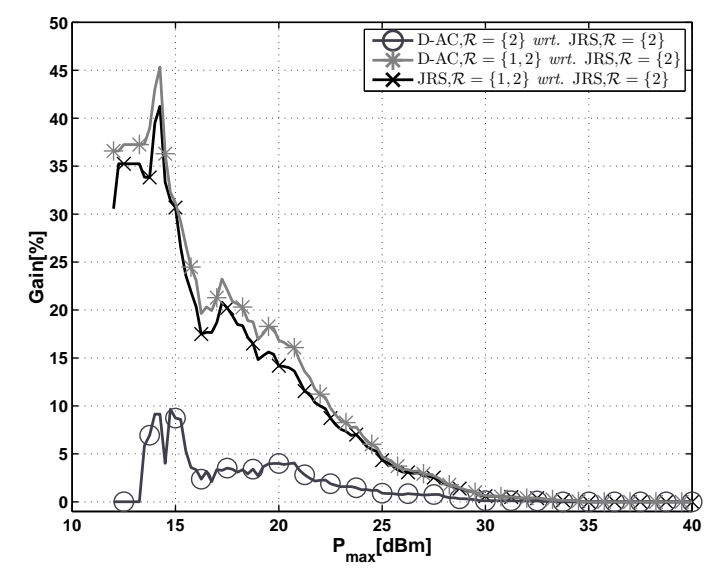

Fig. 5. Relative gains of averaged $R^{*}$ in single-power and multi-rate networks

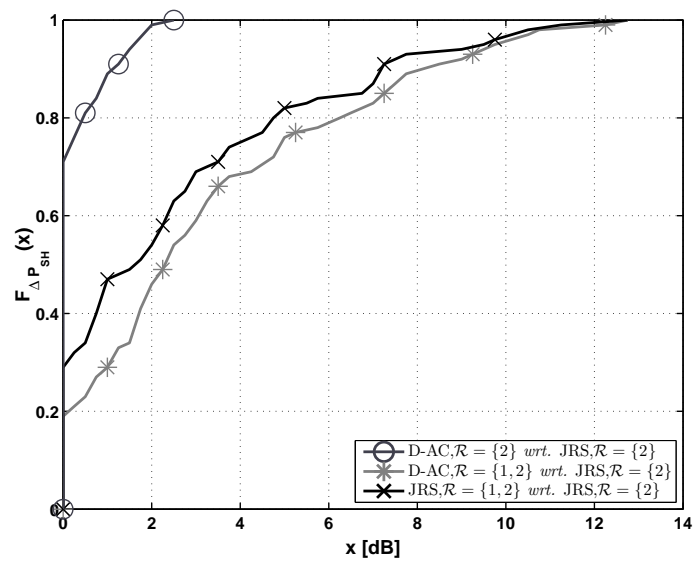

Fig. 6. CDF of $\Delta P_{S H}$ in single-power and multi-rate networks

Multiple rates and single power: Firstly, we study the case when D-AC is enabled in networks where nodes can support the set of rates $\mathcal{R}=\{1,2\}$ but are allowed to transmit only with the maximum transmit power $P_{\max }$. We aim to establish if the multi-rate capability allows D-AC to provide a better throughput gain than the single-rate case. Fig. 5 shows that D-AC can only provide a marginal throughput gain in a multirate network, i.e., there is a small difference of at most $5 \%$ between D-AC, $\mathcal{R}=\{1,2\}$ and JRS, $\mathcal{R}=\{1,2\}$. In fact, Fig. 5 shows that a multi-rate capability can significantly improve throughput (as it was shown before in [14]), while D-AC provides only marginal gains.

Now, we focus on $\Delta P_{S H}$ in multi-rate networks and show in Fig. 6 the empirical CDF of $\Delta P_{S H}$ for the same 3 cases as in Fig. 5. While D-AC, $\mathcal{R}=\{1,2\}$ outperforms JRS, $\mathcal{R}=\{1,2\}$ in terms of $\Delta P_{S H}$, this improvement is very small as it is achieved in only about $10 \%$ of network realizations. When the multi-rate cases of D-AC and JRS are compared with $\mathrm{JRS}, \mathcal{R}=\{2\}$, it is evident that the largest portion of the gain in $\Delta P_{S H}$ is due to the use of multiple rates but not $\mathrm{D}-\mathrm{AC}$ itself. In fact, the gain of D-AC in isolation is very small as indicated by $\mathrm{D}-\mathrm{AC}, \mathcal{R}=\{2\}$ where about $70 \%$ of the realizations do not yield any gain at all and gains above $1 \mathrm{~dB}$ can be achieved in only $10 \%$ of networks.

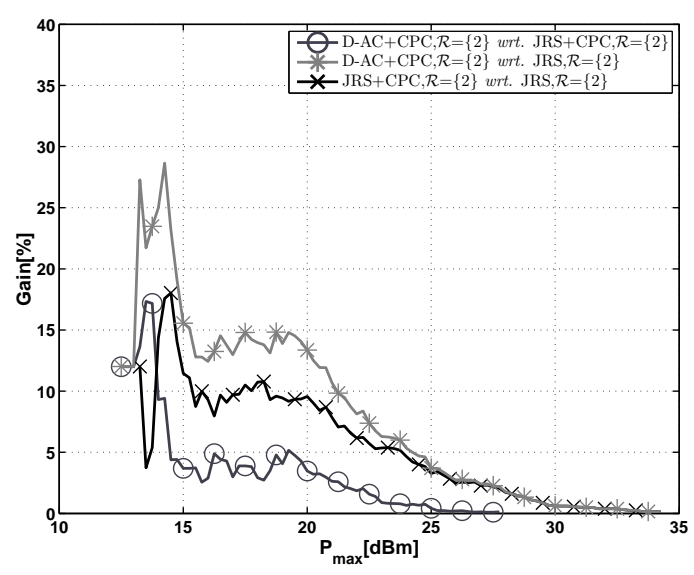

Fig. 7. Relative gains of averaged $R^{*}$ in single-rate networks with CPC

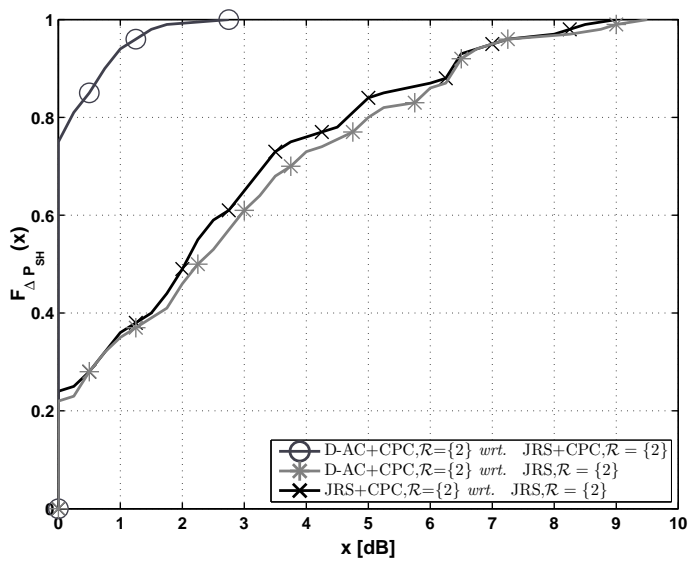

Fig. 8. CDF of $\Delta P_{S H}$ in single-rate networks with CPC

Altogether, the use of D-AC in multi-rate networks does not yield significant gains either in terms of throughput or $P_{S H}$. While one must be careful to recall that such a poor performance of D-AC is only observed for small to medium sized networks and only for networks with 2 available rates, these results do show that D-AC cannot be used as a substitute for multi-rate capability which does provide a significant gain in throughput.

Single rate and continuous power control: In this part, we study the use of D-AC in mesh networks where nodes are enabled with CPC but can only support a single rate so that $\mathcal{R}=\{2\}$. We aim to establish if the use of CPC is an effective technique that can be used with D-AC in order to achieve a better gain in terms of gain $\left(P_{\max }\right)$ or $\Delta P_{S H}$ by combating the increase in interference from virtual antenna arrays.

Fig. 7 shows the metric gain $\left(P_{\max }\right)$ for the max-min throughputs in single-rate networks with CPC. Despite the fact that D-AC with CPC yields a reasonable gain over JRS+CPC of at most $17 \%$ in the low power regime (up to $15 \mathrm{dBm}$ ), overall the gain of D-AC is low and is at most $5 \%$ at medium to high power range for $P_{\max }$. We are careful to draw a conclusion for D-AC with CPC based on the low power regime (near the minimum connectivity power) as this regime is not a typical power range at which a network is operated. To 


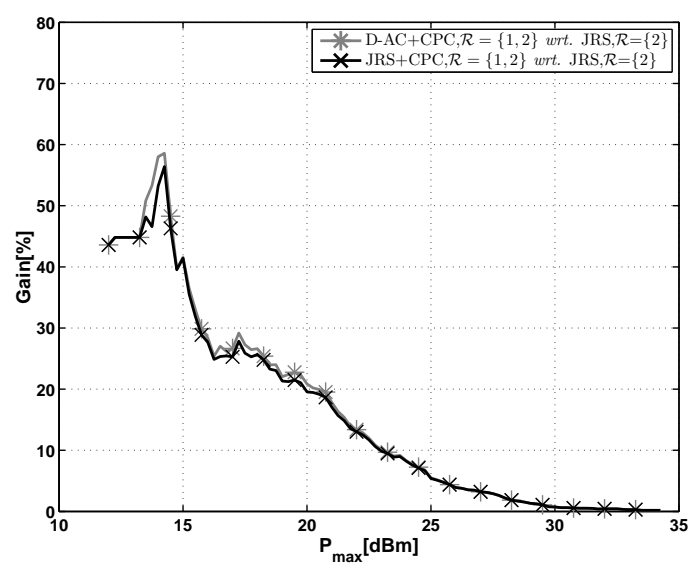

Fig. 9. Relative gains of averaged $R^{*}$ in multi-rate networks with CPC

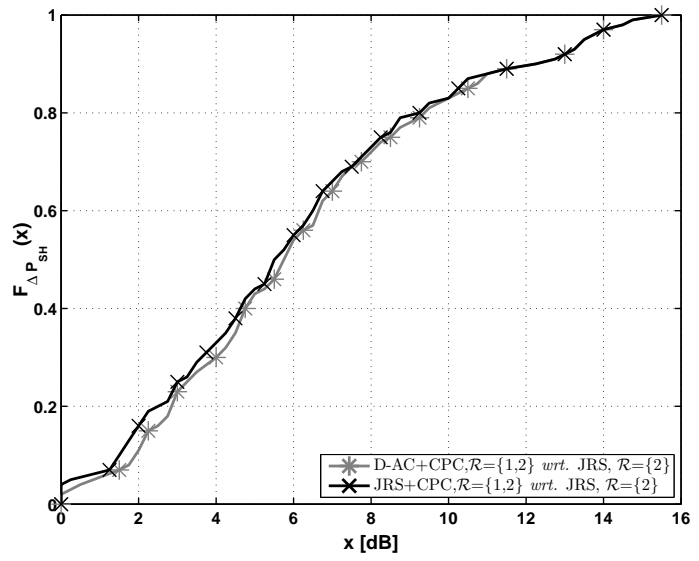

Fig. 10. CDF of $\Delta P_{S H}$ in multi-rate networks with CPC

characterize the impact of $\mathrm{CPC}$ on the gain, let us consider the results for D-AC+CPC and JRS+CPC that are obtained with respect to the baseline results JRS, $\mathcal{R}=\{2\}$. While the case of $\mathrm{D}-\mathrm{AC}+\mathrm{CPC}, \mathcal{R}=\{2\}$ provides a better gain than $\mathrm{JRS}+\mathrm{CPC}, \mathcal{R}=\{2\}$, the improvement is relatively small with only about $5 \%$ difference in gain $\left(P_{\max }\right)$ for medium to high transmit power range. Indeed, the largest portion of the gain in the case of D-AC+CPC can be attributed to the use of CPC alone as $\mathrm{JRS}+\mathrm{CPC}, \mathcal{R}=\{2\}$ provides up to $10 \%$ gain in the medium to high power range and up to $18 \%$ in the low transmit power regime.

Fig. 8 shows the CDFs of $\Delta P_{S H}$ for the same 3 cases. In about $75 \%$ of the network realizations, D-AC with CPC does not provide any gain at all (with respect to JRS+CPC, $\mathcal{R}=$ $\{2\})$ and a gain larger than $1 \mathrm{~dB}$ is obtained in only $10 \%$ of realizations. On the other hand, CPC alone provides a significant gain in $\Delta P_{S H}$ with respect to the results when CPC is not enabled, i.e., in only $21 \%$ of the network realizations, a gain cannot be obtained while a gain of at least $2 \mathrm{~dB}$ and above $6.5 \mathrm{~dB}$ can be provided by CPC in about $50 \%$ and $10 \%$ of realizations, respectively. In effect, the cases of D-AC+CPC and JRS+CPC with respect to JRS, $\mathcal{R}=\{2\}$ indicate that DAC cannot significantly improve the performance of a system with CPC, while CPC is itself an effective technique to provide

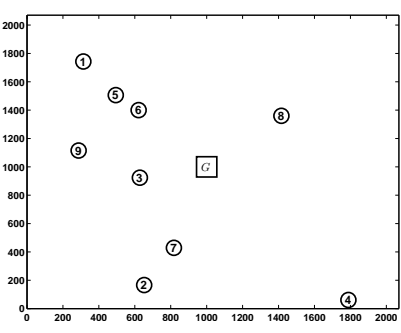

(a) Net-1

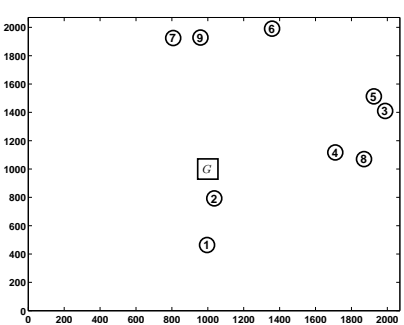

(b) Net-2
Fig. 11. Placement of nodes where $G$ denotes the gateway

a large gain in terms of $\Delta P_{S H}$.

Thus, we conclude that CPC is an efficient technique that can be used to improve max-min throughput and power gain $\Delta P_{S H}$. In the case when CPC is enabled jointly with D-AC, then the largest portion of gain can be attributed to the use of $\mathrm{CPC}$ alone and not to D-AC.

Multiple rates and continuous power control: In this part, we focus on the use of D-AC in networks where nodes are enabled with CPC and can support the set of rates $\mathcal{R}=\{1,2\}$.

Fig. 9 and Fig. 10 show respectively gain $\left(P_{\max }\right)$ and the $\mathrm{CDF}$ of $\Delta P_{S H}$ for both D-AC+CPC and JRS+CPC in multirate networks. Clearly, D-AC provides negligible gains in terms of both metrics Indeed, D-AC itself yields almost no gain in throughput in multi-rate networks with CPC, i.e., the gain at most $3 \%$ in the low power regime for gain $\left(P_{\max }\right)$ when compared with JRS+CPC, $\mathcal{R}=\{1,2\}$, and in only about $2 \%$ of the network realizations is there an improvement in $\Delta P_{S H}$. This indicates that when a network is jointly optimized with only CPC and rate adaptation for the optimal routing and scheduling parameters, then $\mathrm{D}-\mathrm{AC}$ provides almost no gain in such a mesh network. We can also characterize the impact of CPC on the multi-rate capability by comparing with Figs. 5, 6 , and 8 since the results for D-AC+CPC, $\mathcal{R}=\{1,2\}$ include the gain due to the use of multiple rates and CPC. Indeed, CPC in multi-rate networks allows the gain of at most $17 \%$ by comparing the results for $\mathcal{R}=\{1,2\}$ with JRS+CPC and JRS only. This additionally confirms that CPC can effectively control the interference for a better gain in throughput. When CPC or multiple rates are used in isolation, these techniques do not provide as high gains in terms of $\Delta P_{S H}$ as when they are used jointly as shown in Fig. 10. The metric $\Delta P_{S H}$ can be improved in up to $20 \%$ of the network realizations and gains above $10 \mathrm{~dB}$ can be achieved in an additional $10 \%$ of realizations.

Max-min throughput in a given network: In the previous parts, we studied the performance of D-AC and its combination with CPC and multiple rates by characterizing the gains in terms of gain $\left(P_{\max }\right)$ and $\Delta P_{S H}$ over 100 network realizations, yet the particular gains depend on the placement of nodes and channel realizations in a given network. Among the 100 network realizations, we selected two networks, Net-1 and Net-2 shown in Fig. 11, with results that have significantly different outcomes. Fig. 12 and Fig. 13 show the max-min throughput $R^{*}$ as a function of $P_{\max }$ in Net-1 and Net-2, respectively. $P_{S H}$ denotes the minimum transmit power at 


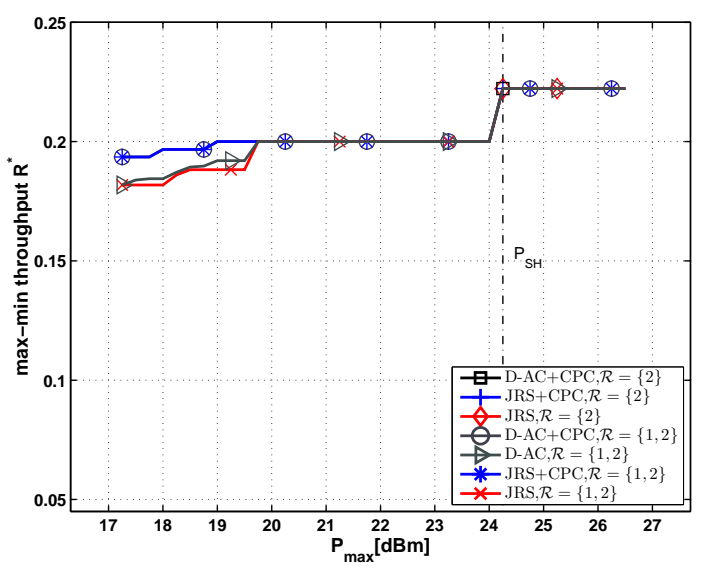

Fig. 12. max-min throughput $R^{*}$ vs $P_{\max }$ in Net-1

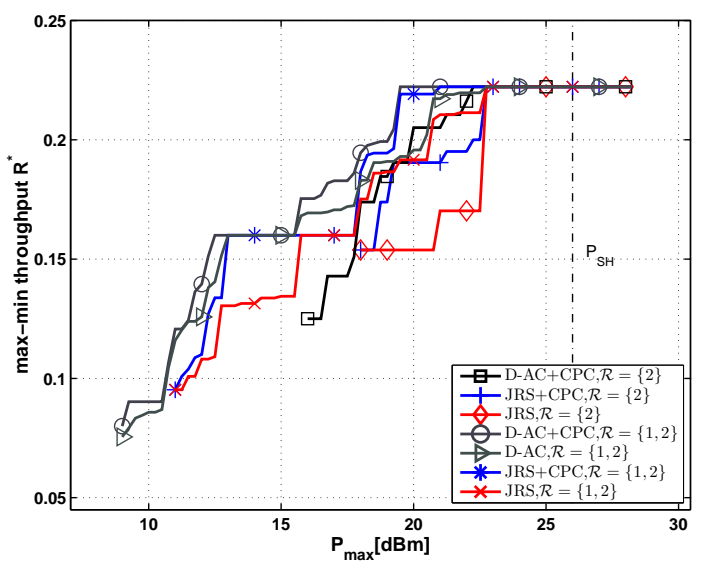

Fig. 13. max-min throughput $R^{*}$ vs $P_{\max }$ in Net-2

which all nodes in the network are able to communicate with the gateway in single hop at rate $r_{m}=2$.

While in Net-2, the use of D-AC+CPC and multi-rate capability yield a significant gain across the whole range of power levels for $P_{\max }$, D-AC in Net-1 provides only a marginal gain and only in the low transmit power regime. With this example, we aim to show that even when a mesh network is throughput-optimally configured, the gains differ significantly from one network topology to another. This example also shows that cooperative relaying can improve significantly the performance in a network where nodes are placed judiciously.

Optimal network configuration: A solution to problem [M] also gives us the optimal network configuration in terms of routing, scheduling, transmit power, and cooperative relaying parameters. Fig. 14 shows an example of the multi-path routing in Net-2 for the uplink flow from node 8 to the gateway along with the amount of flow transmitted over each link and the optimal selection of cooperating node pairs. This routing is optimal and provides the max-min throughput $R=0.08$ for $P_{\max }=15 \mathrm{dBm}$ using D-AC+CPC, $\mathcal{R}=\{1,2\}$. Clearly, the link between the gateway and virtual node $\{2,4\}$ is a bottleneck link, i.e., node $\{2,4\}$ is directly connected to the gateway that needs to delivery the traffic from all the paths.

A note on computation times: On average, we needed less

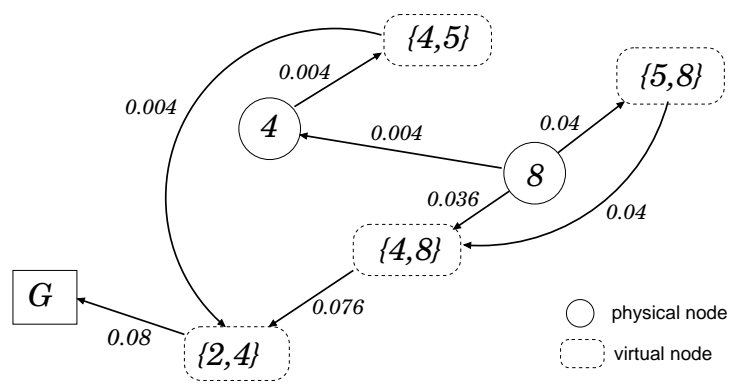

Fig. 14. Illustration of the uplink flow routing from node 8 to gateway $G$ in Net- 2 for the case D-AC+CPC, $\mathcal{R}=\{1,2\}$ and $P_{\max }=15 \mathrm{dBm}$

than two hours per network to obtain the max-min throughput rates for the entire transmit power range with a step size of $0.25 \mathrm{~dB}$. We computed the results using a server with the following specs: 2 CPUs, 12 Cores, $2.93 \mathrm{GHz}$.

\section{CONCLUSION}

In this work, we have established that the use of cooperative relaying based on the Alamouti code does not typically improve significantly the performance of a random mesh network of small to medium size. Even when continuous power control or multi-rate capability is enabled, cooperative relaying often does not yield a significant gain in terms of throughput and connectivity. On the other hand, CPC is proven to be an effective technique to manage interference in conventional multihop networks. These conclusions are drawn from the results that we have obtained for 100 random networks of small to medium size. Note that we have no grounds to generalize these insights to larger mesh networks.

The cross-layer framework that we have proposed in this work is general in that it can be used to obtain the maximum achievable throughput as well as the throughput-optimal configuration of a fixed wireless network that deploys any type of cooperative combining technique. For example, the framework can be directly adopted for multi-relaying and multi-user cellular systems or ad-hoc networks as well as can be easily specialized for distributed beamforming techniques.

\section{REFERENCES}

[1] J. Laneman and G. Wornell, "Distributed space-time-coded protocols for exploiting cooperative diversity in wireless networks," IEEE Trans. Inf. Theory, vol. 49, no. 10, pp. 2415 - 2425, oct. 2003.

[2] A. Sendonaris, A. E. Erkip, and B. Aazhang, "User cooperation diversity - part I: System description,” IEEE Trans. Commun., vol. 51, pp. 1927$1938,2003$.

[3] J. N. Laneman, D. Tse, and G. W. Wornell, "Cooperative diversity in wireless networks: efficient protocols and outage behavior," IEEE Trans. Inform. Theory, vol. 50, pp. 3062-3080, 2004.

[4] G. Kramer, M. Gastpar, and P. Gupta, "Cooperative strategies and capacity theorems for relay networks," IEEE Trans. Inf. Theory, vol. 51, no. 9 , pp. $3037-3063$, Sept. 2005.

[5] K. Jain, J. Padhye, V. N. Padmanabhan, and L. Qiu, "Impact of interference on multi-hop wireless network performance," in Proc. ACM Conf. Mobicom, 2003, pp. 66-80.

[6] A. Karnik, A. Iyer, and C. Rosenberg, "Throughput-optimal configuration of fixed wireless networks," IEEE/ACM Trans. Netw., vol. 16, no. 5, pp. 1161-1174, 2008.

[7] S. Cui and A. Goldsmith, "Cross-layer design of energy-constrained networks using cooperative MIMO techniques," Eurasip's Sig. Proc., vol. 86, pp. 1804-1814, Aug. 2006. 
[8] G. Bo, D. Lin, and L. Cimini, "Routing strategies in multihop cooperative networks," IEEE Trans. Wireless Commun., vol. 8, no. 2, pp. 843 -855 , Feb. 2009.

[9] L. Le and E. Hossain, "Cross-layer optimization frameworks for multihop wireless networks using cooperative diversity," IEEE Trans. Wireless Commun., vol. 7, no. 7, pp. $2592-2602$, July 2008.

[10] A. Iyer, C. Rosenberg, and A. Karnik, "What is the right model for wireless channel interference?" IEEE Trans. Wireless Commun., vol. 8, no. 5, pp. 2662 - 2671, 2009.

[11] S. Sharma, Y. Shi, Y. Hou, H. Sherali, and S. Kompella, "Cooperative communications in multi-hop wireless networks:joint flow routing and relay node assignment," in Proc. IEEE Conf. Infocom, 2010, pp. 1-9.

[12] D. Fooladivanda and C. Rosenberg, "Joint resource allocation and user association for heterogeneous wireless cellular networks," IEEE Trans. Wireless Commun., vol. 12, no. 1, Jan. 2013.

[13] S. Shabdanov, P. Mitran, and C. Rosenberg, "On cooperative wireless relaying: A joint routing and scheduling flow-based framework," in Proc. IEEE Conf. Globecom, 2012, pp. 4641 - 4646.

[14] J. Luo, C. Rosenberg, and A. Girard, "Engineering wireless mesh networks: joint scheduling, routing, power control, and rate adaptation," IEEE/ACM Trans. Netw., vol. 18, pp. 1387-1400, Oct. 2010.

[15] S. Toumpis and A. Goldsmith, "Capacity regions for wireless ad hoc networks," IEEE Trans. Wireless Commun., vol. 2, no. 4, pp. 736 - 748, Jul. 2003.

[16] R. Babaee and N. Beaulieu, "Cross-layer design for multihop wireless relaying networks," IEEE Trans. Wireless Commun., vol. 9, no. 11, 2010.

[17] T. C.-Y. Ng and W. Yu, "Joint optimization of relay strategies and resource allocations in cooperative cellular networks," IEEE Trans. Sel. Commun., vol. 25, no. 2, pp. $328-339$, Febr. 2007.

[18] A. Khandani, J. Abounadi, E. Modiano, and L. Zheng, "Cooperative routing in static wireless networks," IEEE Trans. Wireless Commun., vol. 55, no. 11, pp. $2185-2192$, Nov. 2007.

[19] Z. Guan, T. Melodia, T. Yuan, and D. Pados, "Distributed spectrum management and relay selection in interference-limited cooperative wireless networks," in Proc. ACM Conf. Mobicom, 2011, pp. 229-240.

[20] J. Huang, Z. Han, M. Chiang, and H. V. Poor, "Auction-based resource allocation for cooperative communications," IEEE J.Sel. A. Commun., vol. 26, no. 7, pp. 1226-1237, Sept. 2008.

[21] G. Zhao, C. Yang, G. Y. Li, D. Li, and A. K. Soong, "Power and channel allocation for cooperative relay in cognitive radio networks," IEEE J. Sel. T. Sig. Proc., vol. 5, no. 1, pp. 151 - 159, Jan. 2011.

[22] S. Shabdanov, P. Mitran, and C. Rosenberg, "Cross-layer optimization using advanced physical layer techniques in wireless mesh networks," IEEE Trans. Wireless Commun., vol. 11, no. 4, Apr. 2012.

[23] A. Capone, G. Carello, I. Filippini, S. Gualandi, and F. Malucelli, "Routing, scheduling and channel assignment in wireless mesh networks: optimization models and algorithms," Ad Hoc Netw., vol. 8, no. 6, pp. 545-563, 2010

[24] B. Sethuraman, B. Rajan, and V. Shashidhar, "Full-diversity, high-rate space-time block codes from division algebras," IEEE Trans. Inf. Theory, vol. 49, no. 10, pp. 2596 - 2616, Oct. 2003.

[25] A. Molish, Wireless Communications, 2nd ed. Wiley-IEEE Press, 2005. 